\title{
Estimation on a two-parameter Rayleigh distribution under the progressive Type-Il censoring scheme: comparative study
}

\author{
Jung-In Seo ${ }^{a}$, Byeong-Gyu Seo ${ }^{b}$, Suk-Bok Kang ${ }^{1, b}$ \\ ${ }^{a}$ Department of Statistics, Daejeon University, Korea; \\ ${ }^{b}$ Department of Statistics, Yeungnam University, Korea
}

\begin{abstract}
In this paper, we propose a new estimation method based on a weighted linear regression framework to obtain some estimators for unknown parameters in a two-parameter Rayleigh distribution under a progressive Type-II censoring scheme. We also provide unbiased estimators of the location parameter and scale parameter which have a nuisance parameter, and an estimator based on a pivotal quantity which does not depend on the other parameter. The proposed weighted least square estimator (WLSE) of the location parameter is not dependent on the scale parameter. In addition, the WLSE of the scale parameter is not dependent on the location parameter. The results are compared with the maximum likelihood method and pivot-based estimation method. The assessments and comparisons are done using Monte Carlo simulations and real data analysis. The simulation results show that the estimators $\hat{\mu}_{u}\left(\hat{\theta}_{p}\right)$ and $\hat{\theta}_{p}\left(\hat{\mu}_{u}\right)$ are superior to the other estimators in terms of the mean squared error (MSE) and bias.
\end{abstract}

Keywords: Rayleigh distribution, progressive Type-Il censored sample, weighted linear regression

\section{Introduction}

In statistics, engineering, economics, and medical research, censoring is a condition in which the value of a measurement or observation is only partially known. In life testing experiments or reliability, the observations are often censored. Type-II censoring is the most famous censoring schemes. Type-II censoring occurs if an experiment has a set number of subjects or items and stops the experiment when a predetermined number are observed to have failed; the remaining subjects are then right-censored. But it is not possible to withdraw live units during the experiment. We apply a generalization of the Type-II censoring scheme, where it is possible to withdraw live units during the experiment, and it is well known for progressive Type-II censoring schemes. Progressive Type-II censoring has been suggest in the field of lifetime experiments. Its allowance for the removal of live units from the experiments at various stages is an attractive feature due to its potential to save cost and time for the experimenter.

One of the most popular distributions is the Weibull distribution in lifetime experiments. The Rayleigh distribution can be obtained when the shape parameter of the Weibull distribution is 2 . The Rayleigh distribution was introduced by Rayleigh (1880). The Rayleigh distribution can be used to

\footnotetext{
${ }^{1}$ Corresponding author: Department of Statistics, Yeungnam University, 280 Daehak-ro, Joyeong-dong, Gyeongsan, Gyeongsangbuk-do 38541, Korea. E-mail: sbkang@yu.ac.kr
}

Published 31 March 2019 / journal homepage: http://csam.or.kr

(c) 2019 The Korean Statistical Society, and Korean International Statistical Society. All rights reserved. 
model the lifetime of an object or a service time. The Rayleigh distribution is related to several other distributions such as the chi-squared distribution, extreme value distribution, exponential distribution, normal distribution, Rice distributions and Weibull distribution.

The following are the probability density function (pdf) $f(x)$ and the cumulative distribution function (cdf) $F(x)$ of a random variable $X$ with a two-parameter Rayleigh distribution, location parameter $\mu$, and scale parameter $\theta$ :

$$
\begin{aligned}
& f(x ; \theta, \mu)=2 \theta(x-\mu) e^{-\theta(x-\mu)^{2}}, \quad x>\mu, \theta>0, \\
& F(x ; \theta, \mu)=1-e^{-\theta(x-\mu)^{2}}, \quad x>\mu, \theta>0 .
\end{aligned}
$$

Rayleigh (1880) introduced the Rayleigh distribution, which many studies have since investigated. Johnson et al. (1994) mentioned the two-parameter Rayleigh distribution in their book. Han and Kang (2006) derived approximate maximum likelihood estimators (AMLEs) of the scale parameter in the double Rayleigh distribution. Dey et al. (2014) proposed maximum likelihood estimators (MLEs) and Bayes estimators for a two-parameter Rayleigh distribution based on progressive Type-II censoring with binomial removal. Seo et al. (2016) provided the exact confidence intervals for unknown parameters and exact predictive intervals for future upper record values by providing some pivotal quantities in a two-parameter Rayleigh distribution based on the upper record values. Mkolesia and Shatalov (2017) proposed an exact method for constant minimization of the goal function to estimate parameters of a two-parameter Rayleigh distribution. Fundi et al. (2017) introduced an MLE, EM algorithm, and NR method for a two-parameter Rayleigh distribution based on progressive Type-II censored samples. Seo and Kang (2015) proposed a new method based on a pivotal quantity to estimate the scale parameter of the half logistic distribution based on progressively Type-II censored samples, which provide a simpler estimation equation than the likelihood equation. Seo and Kang (2017) considered estimation and prediction problems for two-parameter half-logistic distribution based on pivotal quantities when a sample is available from a progressively Type-II censoring scheme.

Observations are often censored in experiments for testing lifetime or reliability. Type-I and TypeII censoring are well-known censoring schemes, but live units cannot be withdrawn during the experiment in these schemes. We apply a generalization of the Type-II censoring scheme where it is possible to withdraw live units during the experiment, which is well known for progressive Type-II censoring schemes. This scheme can be explained as follows. Suppose there are $n$ randomly selected units from the Rayleigh distribution with pdf (1.1) in a lifetime test. Suppose that the observation of failures begins at the time of the first failure, and a progressive Type-II censored sample of size $m-1$ is observed as follows. At the time of the first failure, $R_{1}$ surviving units are randomly withdrawn from the test; at the time of the 2nd failure, $R_{2}$ surviving units are randomly withdrawn from the test, and so on. Finally, at the time of the $m$ th failure, all the remaining $R_{m}=n-m-R_{1}-R_{2}-\cdots-R_{m-1}$ are withdrawn from the test. Let $X_{1: m: n} \leq X_{2: m: n} \leq \cdots \leq X_{m: m: n}$ denote such a progressive Type-II censored sample with $\left(R_{1}, \ldots, R_{m}\right)$ being the progressive censoring scheme. Balakrishnan and Aggarwala (2000) provide some historical remarks and a good summary of progressive censoring.

We propose a new approach based on the weighted linear regression framework of unknown parameters in the two-parameter Rayleigh distribution with pdf (1.1) under the progressive Type-II censoring scheme. The rest of the paper is organized as follows. The maximum likelihood method is developed in Section 2, and an estimation method for the pivotal quantity is provided to estimate the location parameter and the scale parameter of the two-parameter Rayleigh distribution under the progressive Type-II censoring scheme. The new estimation method is proposed in Section 3 based on a weighted least-square method with progressive Type-II censored samples. In Section 4, the estimation 
methods considered are assessed and compared using Monte Carlo simulations and real data analysis. Section 5 then concludes the paper.

\section{Classical approach}

This section provides the MLEs and pivot-based estimators of the location parameter $\mu$ and the scale parameter $\theta$ of a two-parameter Rayleigh distribution under the progressive Type-II censoring scheme.

\subsection{Maximum likelihood estimation}

Let $X_{1: m: n}<\cdots<X_{m: m: n}$ be a progressive Type-II censored sample with censoring scheme $\left(R_{1}, \ldots, R_{m}\right)$ from a two-parameter Rayleigh distribution. The corresponding likelihood function is:

$$
\begin{aligned}
L(\mu, \theta) & =c \prod_{i=1}^{m} f\left(x_{i: m: n} ; \theta, \mu\right)\left[1-F\left(x_{i: m: n} ; \theta, \mu\right)\right]^{R_{i}} \\
& =c \prod_{i=1}^{m} 2 \theta\left(x_{i: m: n}-\mu\right) e^{-\left(R_{i}+1\right) \theta\left(x_{i: m: n}-\mu\right)^{2}}, \quad x>\mu, \theta>0,
\end{aligned}
$$

where $c=n\left(n-1-R_{1}\right) \cdots\left(n-R_{1}-\cdots-R_{m-1}-m+1\right)$. From (2.1), the natural logarithm of the likelihood function is:

$$
l(\mu, \theta)=\log L(\mu, \theta)=c_{1} m \ln \theta+\sum_{i=1}^{m} \ln \left(x_{i: m: n}-\mu\right)-\theta \sum_{i=1}^{m}\left(R_{i}+1\right)\left(x_{i: m: n}-\mu\right)^{2},
$$

where $c_{1}$ is a constant. Then, the MLEs of $\mu$ and $\theta, \hat{\mu}$ and $\hat{\theta}$, can be obtained simultaneously by solving the following likelihood equations for $\mu$ and $\theta$ :

$$
\begin{aligned}
& \frac{\partial l(\mu, \theta)}{\partial \theta}=\frac{m}{\theta}-\sum_{i=1}^{m}\left(R_{i}+1\right)\left(x_{i: m: n}-\mu\right)^{2}=0, \\
& \frac{\partial l(\mu, \theta)}{\partial \mu}=-\sum_{i=1}^{m}\left(x_{i: m: n}-\mu\right)^{-1}+2 \theta \sum_{i=1}^{m}\left(R_{i}+1\right)\left(x_{i: m: n}-\mu\right)=0 .
\end{aligned}
$$

\subsection{Estimation based on pivotal quantity}

Based on the pivotal quantity, we apply the method proposed by Seo and Kang $(2016,2017)$ to the two-parameter Rayleigh distribution to obtain the following estimator.

Theorem 1. Suppose that the location parameter $\mu$ is known. Then, an unbiased estimator of the scale parameter $\theta$ is

$$
\hat{\theta}_{p}(\mu)=\frac{m-1}{\sum_{i=1}^{m}\left(R_{i}+1\right)\left(X_{i: m: n}-\mu\right)^{2}} .
$$

Proof: Let

$$
Y_{i: m: n}=-\log \left[1-F\left(X_{i: m: n}\right)\right]=\theta\left(X_{i: m: n}-\mu\right)^{2} .
$$


Then, $Y_{1: m: n}<\cdots<Y_{i: m: n}$ are a progressively Type-II censored sample from the standard exponential distribution. Here, consider the following transformations

$$
\begin{aligned}
S_{1} & =n Y_{1: m: n} \\
S_{i} & =\left[n-\sum_{j=1}^{i-1}\left(R_{j}+1\right)\right]\left(Y_{i: m: n}-Y_{i-1: m: n}\right), \quad i=2, \ldots, m,
\end{aligned}
$$

which are independent and identically distributed from the standard exponential distribution (see Viveros and Balakrishnan, 1994). Wang (2009) found the pivotal quantity which has a $\chi^{2}$ distribution with $2 m$ degrees of freedom:

$$
\begin{aligned}
W_{1}(\mu, \theta) & =2 \sum_{i=1}^{m} S_{i} \\
& =2 \sum_{i=1}^{m}\left(R_{i}+1\right) Y_{i: m: n} \\
& =2 \theta \sum_{i=1}^{m}\left(R_{i}+1\right)\left(X_{i: m: n}-\mu\right)^{2} .
\end{aligned}
$$

The pivotal quantity $W_{1}(\mu, \theta) /(2 m-2)$ converges to one in probability as $m \rightarrow \infty$ because $W_{1}(\mu, \theta)$ has a $\chi^{2}$ distribution with $2 m$ degrees of freedom. Therefore, the unbiased estimator $\hat{\theta}_{p}(\mu)$ is derived as (2.2) from the equation $W_{1}(\mu, \theta)=2(m-1)$, which completes the proof.

Note that $\hat{\theta}_{p}(\mu)$ depends on $\mu$. Now we provide two approaches for estimating $\mu$ : an unbiased estimator based on the first-order statistic and an estimator based on a pivotal quantity, which does not depend on the other parameter $\theta$.

Theorem 2. When $\theta$ is known, an unbiased estimator of $\mu$ based on the first order statistic $X_{1: m: n}$ is :

$$
\hat{\mu}_{u}(\theta)=X_{1: m: n}-\frac{\sqrt{\pi}}{2 \sqrt{n \theta}} .
$$

Proof: Since $\mu$ is always smaller than $X_{1: m: n}$, its expectation can be expressed as:

$$
\mu=E\left(X_{1: m: n}\right)+c,
$$

where $c$ is a negative constant. Here, the pdf of $X_{1: m: n}$ is:

$$
f_{X_{1: m: n}}(x)=2 n \theta(x-\mu) e^{-\theta n(x-\mu)^{2}}, \quad x>\mu .
$$

Therefore, we have

$$
\begin{aligned}
E\left(X_{1: m: n}\right) & =\int_{\mu}^{\infty} 2 n \theta x(x-\mu) e^{-\theta n(x-\mu)^{2}} d x \\
& =2 n \theta \int_{0}^{\infty} t(t+\mu) e^{-\theta n t^{2}} d t \\
& =\int_{0}^{\infty} 2 n \theta t^{2} e^{-\theta n t^{2}} d t+\mu \int_{0}^{\infty} 2 n \theta t e^{-\theta n t^{2}} d t \\
& =\frac{\sqrt{\pi}}{2 \sqrt{n \theta}}+\mu .
\end{aligned}
$$


This completes the proof.

Note that the estimator $\hat{\mu}_{u}(\theta)$ depends on $\theta$. Therefore, the estimator $\hat{\theta}_{p}(\mu)$ can be obtained by solving the following nonlinear equation when $\mu$ is unknown:

$$
\theta=\frac{m-1}{\sum_{i=1}^{m}\left(R_{i}+1\right)\left[x_{i: m: n}-x_{1: m: n}+\sqrt{\pi} /(2 \sqrt{n \theta})\right]^{2}} .
$$

The result is denoted as $\hat{\theta}_{p}\left(\hat{\mu}_{u}\right)$. We can also obtain the estimator $\hat{\mu}_{u}\left(\hat{\theta}_{p}\right)$ by substituting $\theta$ with $\hat{\theta}_{p}\left(\hat{\mu}_{u}\right)$ in the estimator $\hat{\mu}_{u}(\theta)$. The following theorem provides another estimator of $\mu$ that does not depend on the other parameter $\theta$.

We obtain an estimator of the location parameter $\mu$ by applying the two-parameter Rayleigh distribution for the formula introduced by Balakrishnan and Cramer (2014).

Theorem 3. An estimator of $\mu, \hat{\mu}_{p}$, is the solution of the equation $W_{2}(\mu)=2(m-2)$, where

$$
\begin{aligned}
W_{2}(\mu)= & 2(m-1) \log \left[\sum_{j=1}^{m}\left(R_{j}+1\right)\left(X_{j: m: n}-\mu\right)^{2}\right] \\
& -2 \sum_{i=1}^{m-1} \log \left[\sum_{j=1}^{i-1}\left(R_{j}+1\right)\left(X_{j: m: n}-\mu\right)^{2}+\left(n-\sum_{j=1}^{i-1}\left(R_{j}+1\right)\right)\left(X_{i: m: n}-\mu\right)^{2}\right] .
\end{aligned}
$$

Proof: Because $S_{1}, \ldots, S_{m}$ are independent and identically distributed from the standard exponential distribution, we can find the pivotal quantities,

$$
U_{i}=\frac{T_{i}}{T_{m}}, \quad i=1, \ldots, m-1
$$

which are order statistics from the uniform $(0,1)$ distribution with sample size $m-1$, where

$$
T_{i}=\sum_{j=1}^{i} S_{j}=n Y_{1: m: n}+\sum_{j=2}^{i}\left(n-\sum_{k=1}^{j-1}\left(R_{k}+1\right)\right)\left(Y_{j: m: n}-Y_{j-1: m: n}\right) .
$$

Then, from (2.5), we can derive another pivotal quantity,

$$
\begin{aligned}
W_{2}(\mu) & =\sum_{i=1}^{m-1}\left(-2 \log U_{i}\right) \\
& =2 \sum_{i=1}^{m-1} \log \left\{\frac{\sum_{j=1}^{m}\left(R_{j}+1\right) Y_{j: m: n}}{\sum_{j=1}^{i-1}\left(R_{j}+1\right) Y_{j: m: n}+\left[n-\sum_{j=1}^{i-1}\left(R_{j}+1\right)\right] Y_{i: m: n}}\right\}
\end{aligned}
$$

which has a $\chi^{2}$ distribution with $2(m-1)$ degrees of freedom. Therefore, we obtain the equation $W_{2}(\mu)=2(m-2)$ because the pivotal quantity $W_{2}(\mu) /(2 m-4)$ converges to one in probability as $m \rightarrow \infty$ as in the case of the pivotal quantity $W_{1}(\mu, \theta)$. This completes the proof. We denote the estimator $\hat{\theta}_{p}(\mu)$ as $\hat{\theta}_{p}\left(\hat{\mu}_{p}\right)$ by substituting $\mu$ with $\hat{\mu}_{p}$.

Remark 1. The MLE $\hat{\mu}$ and the estimator $\hat{\mu}_{p}$ based on the pivotal quantity $W_{2}(\mu)$ may not be valid because the estimators may have a value larger than $X_{1: m: n}$, while the estimator $\hat{\mu}_{u}\left(\hat{\theta}_{p}\right)$ is admissible. 


\section{Weighted least square estimation}

Lu and Tao (2007) proposed estimation methods based on a linear regression-type framework. We extend the idea to the progressive Type-II censoring scheme and propose new estimators based on a weighted least-square method under the progressive Type-II censoring scheme in this section.

From (2.3), we have

$$
\begin{aligned}
D_{i: m: n} & =Y_{i: m: n}-Y_{1: m: n} \\
& =\theta\left(X_{i: m: n}^{2}-X_{1: m: n}^{2}\right)-2 \theta \mu\left(X_{i: m: n}-X_{1: m: n}\right), \quad i=2, \ldots, m .
\end{aligned}
$$

This leads to the following linear regression:

$$
E\left(D_{i: m: n}\right)=\theta\left(X_{i: m: n}^{2}-X_{1: m: n}^{2}\right)-2 \theta \mu\left(X_{i: m: n}-X_{1: m: n}\right)+\varepsilon_{i}, \quad i=2, \ldots, m,
$$

where $\varepsilon_{i}$ is the error term with $E\left(\varepsilon_{i}\right)=0$. Note that the equation (3.1) can be considered a simple regression line with no intercept. Then, we can obtain new estimators of $\mu$ and $\theta$ by minimizing the following weighted least-square equation under the progressive Type-II censoring scheme:

$$
Q_{w l}=\sum_{i=2}^{m} w_{i: m: n}\left[E\left(D_{i: m: n}\right)-\theta\left(X_{i: m: n}^{2}-X_{1: m: n}^{2}\right)+2 \theta \mu\left(X_{i: m: n}-X_{1: m: n}\right)\right]^{2},
$$

where $w_{i: m: n}$ is the weight of each point, and $E\left(D_{i: m: n}\right)$ was given by Theorem 7.2.1 from Balakrishnan and Cramer (2014):

$$
\begin{aligned}
E\left(D_{i: m: n}\right) & =\sum_{j=2}^{i} \frac{1}{\gamma_{j}}, \quad 2 \leq i \leq m, \\
\gamma_{j} & =\sum_{i=j}^{m}\left(R_{i}+1\right) .
\end{aligned}
$$

The following theorem provides new estimators of $\mu$ and $\theta$ by defining the weight $w_{i: m: n}$.

Theorem 4. Let $w_{i: m: n}=1 / \operatorname{Var}\left(D_{i: m: n}\right)$, where $\operatorname{Var}\left(D_{i: m: n}\right)=\sum_{j=2}^{i} \gamma_{j}^{-2}, 2 \leq i \leq m$ given by Theorem 7.2.1 from Balakrishnan and Cramer (2014). Then, the weighted least-square estimators of $\mu$ and $\theta$ are:

$$
\begin{aligned}
\hat{\mu}_{w l} & =\frac{A_{1}}{2 A_{3}}-\frac{A_{2}\left(A_{3} A_{5}-A_{1}^{2}\right)}{2 A_{3}\left(A_{3} A_{4}-A_{1} A_{2}\right)} \\
\hat{\theta}_{w l} & =\frac{A_{3} A_{4}-A_{1} A_{2}}{A_{3} A_{5}-A_{1}^{2}},
\end{aligned}
$$

respectively, where

$$
\begin{aligned}
& A_{1}=\sum_{i=2}^{m} \frac{\left(X_{i: m: n}^{2}-X_{1: m: n}^{2}\right)\left(X_{i: m: n}-X_{1: m: n}\right)}{\operatorname{Var}\left(D_{i: m: n}\right)}, \\
& A_{2}=\sum_{i=2}^{m} \frac{E\left(D_{i: m: n}\right)\left(X_{i: m: n}-X_{1: m: n}\right)}{\operatorname{Var}\left(D_{i: m: n}\right)}, \\
& A_{3}=\sum_{i=2}^{m} \frac{\left(X_{i: m: n}-X_{1: m: n}\right)^{2}}{\operatorname{Var}\left(D_{i: m: n}\right)}
\end{aligned}
$$




$$
\begin{aligned}
& A_{4}=\sum_{i=2}^{m} \frac{E\left(D_{i: m: n}\right)\left(X_{i: m: n}^{2}-X_{1: m: n}^{2}\right)}{\operatorname{Var}\left(D_{i: m: n}\right)}, \\
& A_{5}=\sum_{i=2}^{m} \frac{\left(X_{i: m: n}^{2}-X_{1: m: n}^{2}\right)^{2}}{\operatorname{Var}\left(D_{i: m: n}\right)} .
\end{aligned}
$$

Proof: Consider the weight $w_{i: m: n}$ which is inversely proportional to the corresponding variance $\operatorname{Var}\left(D_{i: m: n}\right)$, as in the case investigated by Lu and Tao (2007). Then, by differentiating with respect to each parameter in the weighted least-square equation (3.2) with $w_{i: m: n}=1 / \operatorname{Var}\left(D_{i: m: n}\right)$, the corresponding estimation equations for $\mu$ and $\theta$ are:

$$
\begin{aligned}
\frac{\partial Q_{w l}}{\partial \mu}= & 4 \theta \sum_{i=2}^{m} \frac{\left[E\left(D_{i: m: n}\right)-\theta\left(X_{i: m: n}^{2}-X_{1: m: n}^{2}\right)+2 \theta \mu\left(X_{i: m: n}-X_{1: m: n}\right)\right]\left(X_{i: m: n}-X_{1: m: n}\right)}{\operatorname{Var}\left(D_{i: m: n}\right)} \\
= & 0, \\
\frac{\partial Q_{w l}}{\partial \theta}= & -2 \sum_{i=2}^{m} \frac{\left[E\left(D_{i: m: n}\right)-\theta\left(X_{i: m: n}^{2}-X_{1: m: n}^{2}\right)+2 \theta \mu\left(X_{i: m: n}-X_{1: m: n}\right)\right]}{\operatorname{Var}\left(D_{i: m: n}\right)} \\
& \times\left[\left(X_{i: m: n}^{2}-X_{1: m: n}^{2}\right)-2 \mu\left(X_{i: m: n}-X_{1: m: n}\right)\right] \\
= & 0 .
\end{aligned}
$$

In terms of $A_{i}(i=1, \ldots, 5)$, equations (3.3) and (3.4) can be simplified as follows:

$$
\begin{aligned}
& \frac{\partial Q_{w l}}{\partial \mu}=4 \theta\left(A_{2}-A_{1} \theta+2 A_{3} \theta \mu\right)=0, \\
& \frac{\partial Q_{w l}}{\partial \theta}=-2\left(A_{4}-2 A_{2} \mu-A_{5} \theta+4 A_{1} \theta \mu-4 A_{3} \theta \mu^{2}\right)=0 .
\end{aligned}
$$

The proof is completed by solving these equations simultaneously.

Theorem 5. The estimator $\hat{\theta}_{w l}$ is always positive.

Proof: Let

$$
\begin{aligned}
B_{1 i} & =\frac{E\left(D_{i: m: n}\right)\left(X_{i: m: n}-X_{1: m: n}\right)}{\operatorname{Var}\left(D_{i: m: n}\right)}, \\
B_{2 i} & =X_{i: m: n}+X_{1: m: n}, \\
B_{3 i} & =w_{i: m: n}\left(X_{i: m: n}-X_{1: m: n}\right)^{2} .
\end{aligned}
$$

Then, we have

$$
\begin{aligned}
A_{3} A_{4}-A_{1} A_{2} & =\sum_{i=2}^{m} B_{1 i}\left(B_{2 i} \sum_{i=2}^{m} B_{3 i}-\sum_{i=2}^{m} B_{2 i} B_{3 i}\right) \\
& =\sum_{i=2}^{m} B_{1 i}\left(B_{2 i}\left(B_{31}+\cdots+B_{3 m}\right)-\left(B_{21} B_{31}+\cdots+B_{2 m} B_{3 m}\right)\right) \\
& =B_{12} B_{22} B_{31}+B_{13} B_{23} B_{31}+B_{13} B_{23} B_{32}+\cdots+B_{1 m} B_{2 m} B_{3 m-1} \\
& >0 .
\end{aligned}
$$


Table 1: The mean squared errors (biases) of the estimators of $\mu$

\begin{tabular}{|c|c|c|c|c|c|c|}
\hline$n$ & $m$ & Scheme & $\hat{\mu}$ & $\hat{\mu}_{u}\left(\hat{\theta}_{p}\right)$ & $\hat{\mu}_{p}$ & $\hat{\mu}_{w l}$ \\
\hline \multirow{8}{*}{20} & \multirow{4}{*}{18} & I & $0.02188(0.11612)$ & $0.01550(-0.00551)$ & $0.02277(0.12684)$ & $0.03788(0.16587)$ \\
\hline & & II & $0.02173(0.11571)$ & $0.01515(-0.00532)$ & $0.02282(0.12669)$ & $0.03843(0.16726)$ \\
\hline & & III & $0.02204(0.11626)$ & $0.01533(-0.00541)$ & $0.02284(0.12685)$ & $0.03818(0.16655)$ \\
\hline & & IV & $0.02173(0.11578)$ & $0.01537(-0.00550)$ & $0.02263(0.12630)$ & $0.03820(0.16662)$ \\
\hline & \multirow{4}{*}{14} & I & $0.02471(0.12162)$ & $0.01706(-0.00779)$ & $0.02352(0.12936)$ & $0.03913(0.16915)$ \\
\hline & & II & $0.02373(0.11928)$ & $0.01581(-0.00697)$ & $0.02341(0.12861)$ & $0.03954(0.17015)$ \\
\hline & & III & $0.02478(0.12148)$ & $0.01649(-0.00739)$ & $0.02355(0.12918)$ & $0.03916(0.16925)$ \\
\hline & & IV & $0.02429(0.12040)$ & $0.01658(-0.00774)$ & $0.02298(0.12780)$ & $0.03889(0.16812)$ \\
\hline \multirow{8}{*}{30} & \multirow{4}{*}{26} & $\mathrm{I}$ & $0.01318(0.09163)$ & $0.00993(-0.00327)$ & $0.01513(0.10294)$ & $0.02493(0.13394)$ \\
\hline & & II & $0.01305(0.09126)$ & $0.00968(-0.00314)$ & $0.01517(0.10290)$ & $0.02541(0.13527)$ \\
\hline & & III & $0.01336(0.09189)$ & $0.00981(-0.00321)$ & $0.01516(0.10297)$ & $0.02512(0.13454)$ \\
\hline & & IV & $0.01309(0.09125)$ & $0.00984(-0.00328)$ & $0.01496(0.10236)$ & $0.02513(0.13421)$ \\
\hline & \multirow{4}{*}{18} & I & $0.01466(0.09518)$ & $0.01053(-0.00456)$ & $0.01536(0.10424)$ & $0.02544(0.13620)$ \\
\hline & & II & $0.01408(0.09346)$ & $0.00969(-0.00393)$ & $0.01539(0.10391)$ & $0.02620(0.13869)$ \\
\hline & & III & $0.01480(0.09518)$ & $0.01012(-0.00425)$ & $0.01545(0.10427)$ & $0.02563(0.13698)$ \\
\hline & & IV & $0.01412(0.09366)$ & $0.01020(-0.00454)$ & $0.01499(0.10256)$ & $0.02530(0.13526)$ \\
\hline \multirow{12}{*}{40} & \multirow{4}{*}{36} & I & $0.00958(0.07857)$ & $0.00720(-0.00055)$ & $0.01152(0.08997)$ & $0.01850(0.11451)$ \\
\hline & & II & $0.00942(0.07815)$ & $0.00708(-0.00050)$ & $0.01150(0.08991)$ & $0.01876(0.11540)$ \\
\hline & & III & $0.00936(0.07800)$ & $0.00714(-0.00053)$ & $0.01151(0.08994)$ & $0.01861(0.11498)$ \\
\hline & & IV & $0.00939(0.07809)$ & $0.00716(-0.00056)$ & $0.01144(0.08959)$ & $0.01867(0.11502)$ \\
\hline & \multirow{4}{*}{28} & I & $0.00951(0.07808)$ & $0.00732(-0.00257)$ & $0.01162(0.08943)$ & $0.01876(0.11604)$ \\
\hline & & II & $0.00939(0.07744)$ & $0.00708(-0.00233)$ & $0.01152(0.08961)$ & $0.01925(0.11794)$ \\
\hline & & III & $0.00965(0.07805)$ & $0.00714(-0.00245)$ & $0.01167(0.08954)$ & $0.01893(0.11675)$ \\
\hline & & IV & $0.00932(0.07739)$ & $0.00719(-0.00257)$ & $0.01161(0.08846)$ & $0.01880(0.11608)$ \\
\hline & \multirow{4}{*}{16} & $\mathrm{I}$ & $0.01157(0.08391)$ & $0.00837(-0.00489)$ & $0.01174(0.09027)$ & $0.01963(0.11965)$ \\
\hline & & II & $0.01069(0.08131)$ & $0.00718(-0.00388)$ & $0.01160(0.08994)$ & $0.02033(0.12252)$ \\
\hline & & III & $0.01168(0.08383)$ & $0.00786(-0.00440)$ & $0.01172(0.09046)$ & $0.01983(0.12053)$ \\
\hline & & IV & $0.01091(0.08189)$ & $0.00793(-0.00483)$ & $0.01175(0.08814)$ & $0.01912(0.11739)$ \\
\hline
\end{tabular}

This indicates that the numerator of the estimator $\hat{\theta}_{w l}$ is positive. In addition, the denominator is also positive because

$$
A_{3} A_{5}=A_{1}^{2}+C
$$

where

$$
\begin{aligned}
C= & w_{2: m: n} w_{3: m: n}\left(X_{2: m: n}-X_{1: m: n}\right)^{2}\left(X_{3: m: n}^{2}-X_{1: m: n}^{2}\right)^{2}+\cdots \\
& +w_{m-1: m: n} w_{m: m: n}\left(X_{m-1: m: n}-X_{1: m: n}\right)^{2}\left(X_{m: m: n}^{2}-X_{1: m: n}^{2}\right)^{2} .
\end{aligned}
$$

This completes the proof.

Remark 2. The estimator $\hat{\mu}_{w l}$ may not be valid, as in the case of $\hat{\mu}$ and $\hat{\mu}_{p}$.

\section{Application}

This section examines the validity of the proposed method through Monte Carlo simulations and real data analysis.

\subsection{Simulation study}

To assess and compare the discussed estimators in the Sections 2 and 3, we report their mean squared errors (MSEs) and biases in Tables 1 and 2. The progressive Type-II censored samples are generated 
Table 2: The mean squared errors (biases) of the estimators of $\theta$

\begin{tabular}{|c|c|c|c|c|c|c|}
\hline$n$ & $m$ & Scheme & $\widehat{\theta}$ & $\hat{\theta}_{p}\left(\hat{\mu}_{u}\right)$ & $\hat{\theta}_{p}\left(\hat{\mu}_{p}\right)$ & $\widehat{\theta}_{w l}$ \\
\hline \multirow{8}{*}{20} & \multirow{4}{*}{18} & $\mathrm{I}$ & $0.46591(0.39721)$ & $0.16682(0.04204)$ & $0.28832(0.32407)$ & $0.35769(-0.05326)$ \\
\hline & & II & $0.40350(0.36992)$ & $0.15326(0.03805)$ & $0.26142(0.29992)$ & $0.30091(-0.07389)$ \\
\hline & & III & $0.44619(0.38687)$ & $0.16022(0.04013)$ & $0.27668(0.31295)$ & $0.33033(-0.05876)$ \\
\hline & & IV & $0.44897(0.38742)$ & $0.16065(0.03965)$ & $0.27532(0.31256)$ & $0.30707(-0.07153)$ \\
\hline & \multirow{4}{*}{14} & I & $1.11865(0.56512)$ & $0.30318(0.06844)$ & $0.53712(0.42310)$ & $0.76992(-0.07398)$ \\
\hline & & II & $0.67353(0.44613)$ & $0.22639(0.05125)$ & $0.37798(0.33128)$ & $0.45654(-0.08379)$ \\
\hline & & III & $0.91483(0.51607)$ & $0.26586(0.06048)$ & $0.48177(0.38259)$ & $0.62273(-0.06572)$ \\
\hline & & IV & $0.90011(0.51375)$ & $0.26546(0.05788)$ & $0.45264(0.37803)$ & $0.49526(-0.06645)$ \\
\hline \multirow{8}{*}{30} & \multirow{4}{*}{26} & I & $0.21751(0.28219)$ & $0.10272(0.02802)$ & $0.17030(0.25442)$ & $0.20453(-0.03156)$ \\
\hline & & II & $0.18717(0.25844)$ & $0.09252(0.02451)$ & $0.14841(0.22962)$ & $0.17011(-0.05319)$ \\
\hline & & III & $0.20705(0.27286)$ & $0.09777(0.02635)$ & $0.16004(0.24284)$ & $0.18812(-0.03635)$ \\
\hline & & IV & $0.20501(0.27276)$ & $0.09841(0.02598)$ & $0.15904(0.24317)$ & $0.17455(-0.05241)$ \\
\hline & \multirow{4}{*}{18} & I & $0.52673(0.42172)$ & $0.18040(0.04666)$ & $0.32749(0.34999)$ & $0.46649(-0.05621)$ \\
\hline & & II & $0.30468(0.31619)$ & $0.12977(0.03214)$ & $0.21060(0.25289)$ & $0.26352(-0.07190)$ \\
\hline & & III & $0.42688(0.37807)$ & $0.15587(0.03995)$ & $0.27173(0.30692)$ & $0.37518(-0.04926)$ \\
\hline & & IV & $0.42156(0.37678)$ & $0.15728(0.03786)$ & $0.26358(0.30484)$ & $0.28694(-0.05830)$ \\
\hline \multirow{12}{*}{40} & \multirow{4}{*}{36} & I & $0.12106(0.21567)$ & $0.06645(0.02061)$ & $0.10970(0.20719)$ & $0.12364(-0.02572)$ \\
\hline & & II & $0.10917(0.20138)$ & $0.06162(0.01864)$ & $0.09862(0.19164)$ & $0.11005(-0.04145)$ \\
\hline & & III & $0.11407(0.20761)$ & $0.06410(0.01967)$ & $0.10425(0.19973)$ & $0.11697(-0.02993)$ \\
\hline & & IV & $0.11516(0.20943)$ & $0.06452(0.01952)$ & $0.10472(0.20058)$ & $0.11211(-0.04146)$ \\
\hline & \multirow{4}{*}{28} & I & $0.19497(0.26893)$ & $0.09593(0.02673)$ & $0.16612(0.25335)$ & $0.20679(-0.02887)$ \\
\hline & & II & $0.14259(0.22120)$ & $0.07663(0.02011)$ & $0.12286(0.20122)$ & $0.14451(-0.05070)$ \\
\hline & & III & $0.17279(0.24851)$ & $0.08657(0.02363)$ & $0.14572(0.22955)$ & $0.17911(-0.02910)$ \\
\hline & & IV & $0.17107(0.25031)$ & $0.08804(0.02289)$ & $0.14529(0.23122)$ & $0.15269(-0.04846)$ \\
\hline & \multirow{4}{*}{6} & I & $0.76254(0.49684)$ & $0.22886(0.05820)$ & $0.42622(0.39723)$ & $0.68288(-0.08647)$ \\
\hline & & II & $0.29968(0.30785)$ & $0.13433(0.03255)$ & $0.20786(0.23311)$ & $0.28973(-0.07801)$ \\
\hline & & III & $0.54592(0.42113)$ & $0.18293(0.04656)$ & $0.32679(0.32876)$ & $0.50467(-0.06786)$ \\
\hline & & IV & $0.50642(0.41166)$ & $0.17992(0.04134)$ & $0.29847(0.31805)$ & $0.32605(-0.04793)$ \\
\hline
\end{tabular}

from a two-parameter Rayleigh distribution with $\mu=0$ and $\theta=1$ for following censoring schemes:

Scheme I : $R_{m}=n-m$ and $R_{i}=0$ for $i \neq m$,

Scheme II $: R_{1}=n-m$ and $R_{i}=0$ for $i \neq 1$,

Scheme III $: R_{1}=R_{m}=\frac{n-m}{2}$ and $R_{i}=0$ for $i \neq 1$ and $m$,

Scheme IV $: R_{m / 2}=n-m$ and $R_{i}=0$ for $i \neq \frac{m}{2}$.

As mentioned, the estimators $\hat{\mu}, \hat{\mu}_{p}$, and $\hat{\mu}_{w l}$ may have a larger value than $X_{1: m: n}$. In this case, $X_{1: m: n}$ is used as an estimate. All MSEs and biases are obtained over 10,000 simulations.

Tables 1 and 2 show that, the estimator $\hat{\mu}_{u}\left(\hat{\theta}_{p}\right)$ is more efficient than other estimators for $\mu$, and the estimator $\hat{\theta}_{p}\left(\hat{\mu}_{u}\right)$ is more efficient than other estimators for $\theta$ in terms of the MSE and bias. In particular, it is notable that the biases of $\hat{\mu}_{u}\left(\hat{\theta}_{p}\right)$ are close to zero, even if the sample size is small. As expected, the MSEs of all estimators increase generally as the number of unobserved or missing data $n-m$ increases for fixed sample size $n$ and the MSEs decrease as sample size $n$ increases.

\subsection{Real data}

We consider a real strength dataset that was originally reported by Badar and Priest (1982). The dataset includes the strength measured in GPA for single carbon fibers and impregnated 1000-carbon fiber tows. Dey et al. (2011) showed that the two-parameter Rayleigh distribution fits the data quite 
Table 3: Observed progressive Type-II censored sample

\begin{tabular}{llllllllll}
\hline \hline 0.562 & 0.564 & 0.729 & 0.802 & 0.950 & 1.053 & 1.111 & 1.115 & 1.194 & 1.208 \\
1.247 & 1.256 & 1.271 & 1.277 & 1.348 & 1.390 & 1.429 & 1.474 & 1.503 & 1.520 \\
1.524 & 1.551 & 1.551 & 1.609 & 1.632 & & & & & \\
\hline \hline
\end{tabular}

Table 4: Estimates of $\mu$ and $\theta$ for the observed progressive Type-II censored sample

\begin{tabular}{cccccccc}
\hline \hline$\hat{\mu}$ & $\hat{\mu}_{u}\left(\hat{\theta}_{p}\right)$ & $\hat{\mu}_{p}$ & $\hat{\mu}_{w l}$ & $\hat{\theta}$ & $\hat{\theta}_{p}\left(\hat{\mu}_{u}\right)$ & $\hat{\theta}_{p}\left(\hat{\mu}_{p}\right)$ & $\hat{\theta}_{w l}$ \\
\hline 0.455 & 0.378 & 0.471 & 0.562 & 0.407 & 0.337 & 0.403 & 0.495 \\
\hline \hline
\end{tabular}

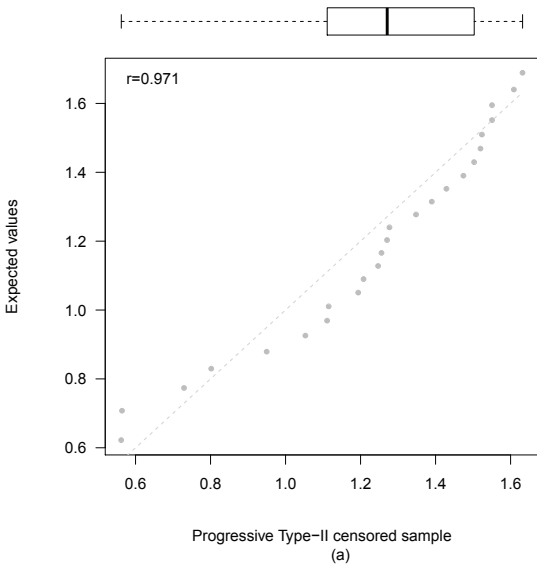

(a)

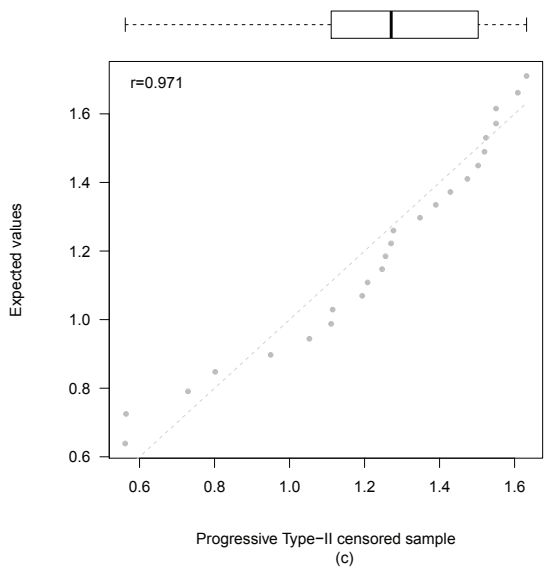

(c)

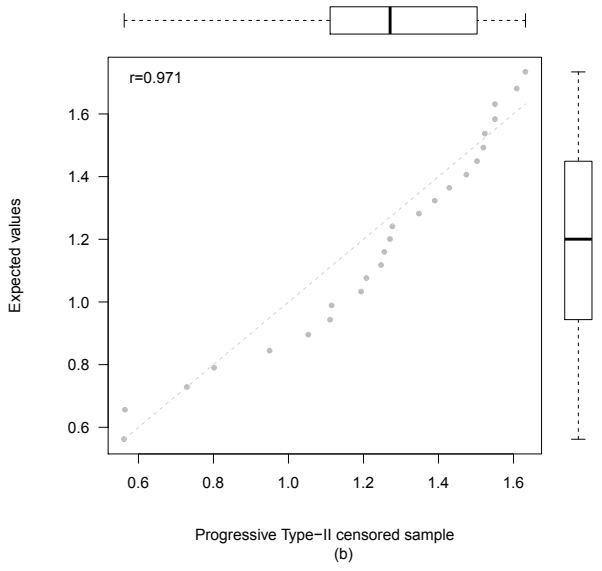

(b)

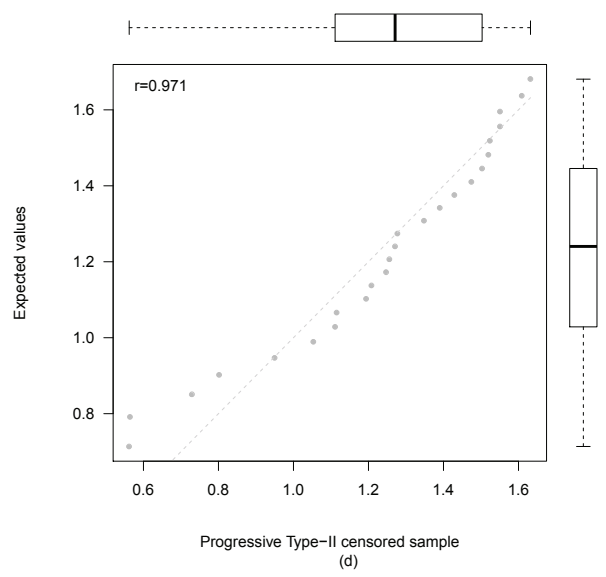

(d)

Figure 1: Boxplots and scatterplots between the observed progressive Type-II censored samples and expected values from (a) $f_{X_{i: m: n}}(x ; \hat{\mu}, \hat{\theta})$; (b) $f_{X_{i: m: n}}\left(x ; \hat{\mu}_{u}\left(\hat{\theta}_{p}\right), \hat{\theta}_{p}\left(\hat{\mu}_{u}\right)\right)$; (c) $f_{X_{i: m: n}}\left(x ; \hat{\mu}_{p}, \hat{\theta}_{p}\left(\hat{\mu}_{p}\right)\right) ;(d) f_{X_{i: m: n}}\left(x ; \hat{\mu}_{w l}, \hat{\theta}_{w l}\right)$.

well. For the analysis, we use a progressive Type-II censored sample with the censoring scheme $(24 * 1,20)$ from the real data, which was reported by Dey et al. (2015). These values are given in Table 3. For the assessment of the goodness of fit of the two-parameter Rayleigh distribution, we first compute the expected values from the estimated marginal density function $f_{X_{i: m: n}}(x ; \tilde{\mu}, \tilde{\theta})$. We then 
plot the boxplot and scatterplot with the correlation coefficient (r) between the observed progressive Type-II censored sample and all expected values (Figure 1). The estimates are reported in Table 4.

Table 4 shows that $\hat{\mu}_{w l}$ is out of the range of the parameter $\mu$ for the observed progressive Type-II censored sample because $\hat{\mu}_{w l}=x_{1: m: n}$. Figure 1 shows a strong correlation between the observed a progressive Type-II censored sample and the expected values from all the estimated marginal density functions, which indicates that the two-parameter Rayleigh distribution fits a progressive Type-II censored sample.

\section{Concluding remarks}

This paper proposed a new estimation method based on a weighted linear regression framework to estimate the unknown parameters of a two-parameter Rayleigh distribution under the progressive Type-II censoring scheme. The proposed method was compared with the maximum likelihood method and an estimation method based on the pivotal quantity. In addition, we provided an unbiased estimator of the location parameter, which has a nuisance parameter as a scale parameter but is admissible, unlike the other estimators considered. The simulation results showed that the unbiased estimator-based $\hat{\mu}_{u}\left(\hat{\theta}_{p}\right)$ and $\hat{\theta}_{p}\left(\hat{\mu}_{u}\right)$ are superior to the other estimators considered in terms of the MSE and bias. Therefore, it is recommended that these estimators be used for the Rayleigh distribution with pdf (1.1) under the progressive Type-II censoring scheme.

The weighted least square estimator (WLSE) of the location parameter is not dependent on the scale parameter. In addition, the WLSE of the scale parameter is also not dependent on the location parameter (Theorem 4). The WLSE of the scale parameter is always greater than 0 . Therefore the range of the scale parameter is always satisfied (Theorem 5).

\section{References}

Bader MG and Priest AM (1982). Statistical aspects of fiber and bundle strength in hybrid composites. In Hayashi T, Kawata K, and Umekawa S (Eds), Progressive in Science and Engineering Composites, (pp. 1129-1136), Tokyo.

Balakrishnan N and Aggarwala R (2000). Progressive Censoring: Theory, Methods, and Applications, Birkhäuser, Boston.

Balakrishnan N and Cramer E (2014). The Art of Progressive Censoring, Springer, New York.

Dey S, Dey T, and Kundu D (2014). Two-parameter Rayleigh distribution: different methods of estimation, American Journal of Mathematical and Management Sciences, 33, 55-74.

Dey T, Dey S, and Kundu D (2015). On progressively Type-II censored two-parameter Rayleigh distribution, Communications in Statistics - Simulation and Computation, 45, 438-455.

Fundi MD, Njenga EG, and Keitany KG (2017) Estimation of parameters of the two-parameter Rayleigh distribution based on progressive Type-II censoring using maximum likelihood method via the NR and the EM algorithms, American Journal of Theoretical and Applied Statistics, 6 , $1-9$.

Han JT and Kang SB (2006). Estimation for the double Rayleigh distribution based on multiply TypeII censored sample, Journal of the Korean Data and Information Science Society, 17, 1319-1328.

Johnson N, Kotz S, and Balakrishnan N (1994) Continuous Univariate Distribution, Wiley, New York.

Lu HL and Tao SH (2007). The estimation of Pareto distribution by a weighted least square method, Quality \& Quantity, 41, 913-926.

Mkolesia AC and Shatalov MY (2017). Exact solutions for a two-parameter Rayleigh distribution, Global Journal of Pure and Applied Mathematics, 13, 8039-8051. 
Rayleigh JWS (1880). On the resultant of a large number of vibrations of the some pitch and of arbitrary phase, Philosophical Magazine, 5-th Series, 10, 73-78.

Seo JI, Jeon JW, and Kang SB (2016). Exact interval inference for the two-parameter Rayleigh distribution based on the upper record values, Journal of Probability and Statistics, 2016, 1-5.

Seo JI and Kang SB (2015). Pivotal inference for the scaled half logistic distribution based on progressively Type-II censored samples, Statistics and Probability Letters, 104, 109-116.

Seo JI and Kang SB (2016). More efficient approaches to the exponentiated half-logistic distribution based on record values, SpringerPlus, 5, 1433.

Seo JI and Kang SB (2017). Inference for the two-parameter half logistic distribution using pivotal quantities under progressively Type-II censoring schemes, Communications in StatisticsSimulation and Computation, 46, 5462-5478.

Viveros R and Balakrishnan N (1994). Interval estimation of parameters of life from progressively censored data, Technometrics, 36, 84-91.

Wang B (2009). Interval estimation for the scaled half-logistic distribution under progressive Type-II censoring, Communications in Statistics: Theory and Methods, 38, 364-371. 